The passerine specimens consist of a proximal right carpometacarpus (Queensland Museum F20688) and distal right tibiotarsus (QM F24685), comparable in size to a grassfinch (Estrildidae) and thrush (Turdus), respectively, indicating that they represent two taxa. Although the proximal carpometacarpal portion is fragmentary ( $a$ in the figure), it exhibits a large intermetacarpal process, a structure found in the Passeriformes and only a limited number of other avian orders: Galliformes, Coliiformes, Coraciiformes and Piciformes. From these the Murgon carpometacarpus is separated and identified as belonging to the Passeriformes by a combination of the shapes of the rims of the carpal trochlea, position and caudal extension of the intermetacarpal process, position of the pisiform process and the condition of the area between the extensor and pisiform processes. The tibio tarsus ( $b$ in the figure) is broken through the shaft; the distal portion has been repaired but is largely complete. This specimen is allocated to the Passeriformes by the condition of the lateral and medial margins of the shaft, and the shapes and positions of lateral and medial condyles, supratendinal bridge and anterior intercondylar fossa. A more detailed presentation of the diagnostic characters of the Passeriformes for these skeletal elements will appear elsewhere.

These are the oldest known passerines
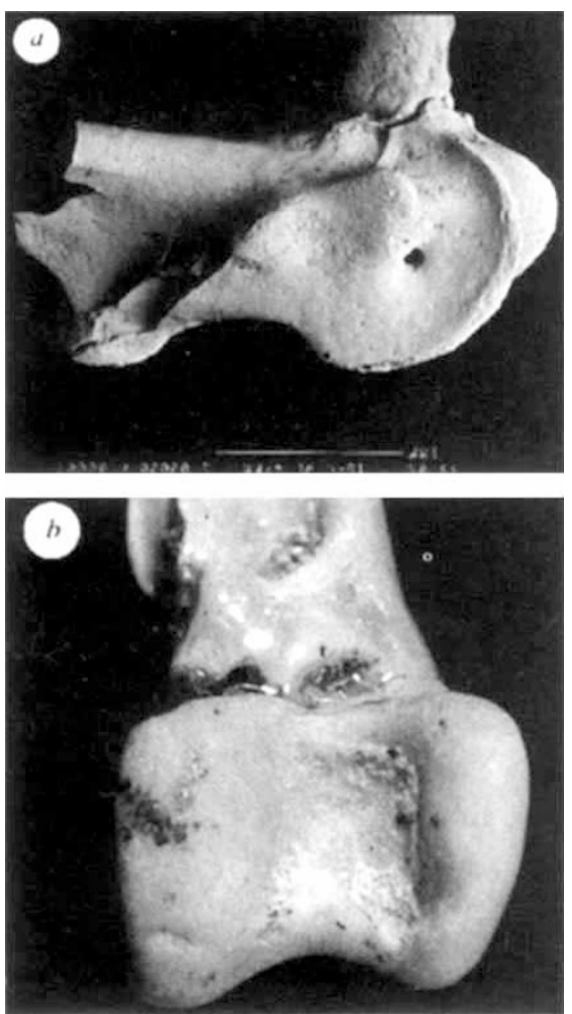

Passerine remains from Early Eocene deposits at Murgon, Queensland; a, proximal carpometacarpal fragment (QM F20688), ventral view (line equals $1 \mathrm{~mm}$ ); $b$, distal tibiotarsal fragment (QM F24685), cranial view (distal width $4.1 \mathrm{~mm})$. by almost 25 million years. They are consistent with a hypothesis of a southern origin for passerines. Little more can be discerned about the relationships of these fossils, nor can a palaeoenvironmental interpretation be made on the basis of their presence.

Walter E. Boles

Division of Vertebrate Zoology (Birds), Australian Museum, 6 College Street, Sydney, New South Wales 2000, Australia

\section{Dorsoventral axis inversion?}

SIR - Arendt and Nübler-Jung in Scientific Correspondence ${ }^{1}$ point out that the decapentaplegic gene is expressed ventrally in the fruitfly Drosophila, whereas the homologous gene $B M P-4$ is expressed dorsally in vertebrates. They explain this paradox by supposing that an ancestor of vertebrates was bodily inverted in evolution, as long ago suggested ${ }^{2}$.

Their suggestion is not compatible with the calcichordate theory of chordate origins $^{3-6}$ (see figure). This theory implies that echinoderms and chordates are both descended from an ancestor like the extant pterobranch Cephalodiscus which lay right-side-downwards on the sea floor (dexiothetism), so that old right became new ventral. Subsequently, in the stem lineage of the chordates, the tail (stalk) rotated through $90^{\circ}$ anticlockwise when seen from behind (counter-torsion of hind tail) which nullified dexiothetism in the tail. Counter-torsion did not affect the head. Instead there was later, in the head, a mirror-duplication on the right of organs (gill slits, left pharynx) which had previously existed only on the left. This organ-pairing still occurs in the metamorphosis of amphioxus.

Arendt and Nübler-Jung's paradox concerns organs derived from the calcichordate tail, where, because dexiothetism has been nullified by counter-torsion, chordate dorsal ought to be equivalent to insect dorsal. To explain it we suggest that chordate neurulation is a late phase of gastrulation. For in amphioxus and the tunicates the blastopore shifts forward during neurulation to become the neuropore at the anterior end of the animal while the internal cavity of the dorsal nerve cord long remains continuous with the archenteron by the neurenteric canal. We suggest that the expression pattern of decapentaplegic and its homologues may depend, not on inversion of the whole animal, but on the position of the gastrulation-neurulation process, being dorsal in chordates and ventral in insects.

Lacalli's suggestion ${ }^{7}$ that the chordate central nervous system originated by downward folding of dorsal ectoderm in an echinoderm-type larva is compatible

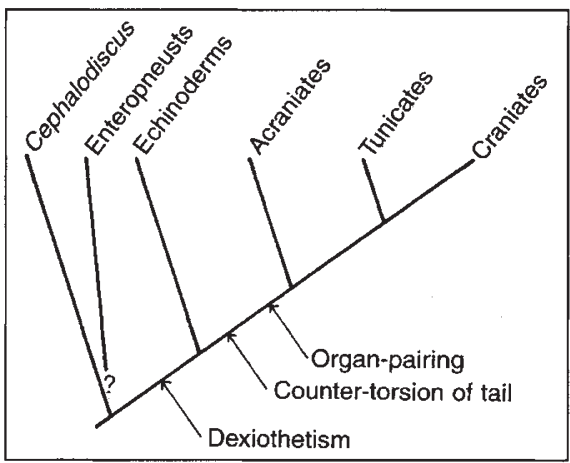

Phylogeny of deuterostomes according to the calcichordate theory with the successive origins of dexiothetism, counter-torsion of the tail and organ-pairing in the head. For further discussion see ref. 6. Amphioxus is an acraniate. The group Craniata here comprises Myxinoida and Vertebrata (lampreys + gnathostomes).

with this explanation. In the calcichordatehead-derived parts of chordates, which were affected by dexiothetism but not by counter-torsion, the homologues of members of the dorsal cascade of Drosophila will probably control left-right rather than ventrodorsal pattern 6 .

Peterson $^{8}$ argues, with good reason, that the ventral nervous system of enteropneusts is homologous to the central nervous system of insects. He also contends, however, that the enteropneust dorsal nervous system is homologous with the dorsal nervous system of chordates. This is doubtful, as the fossils indicate that the chordate central nervous system arose in the tail, with the brain situated where the tail joins the head. Adult enteropneusts have nothing corresponding to the tail. All parts of their nervous system are therefore too anterior to be homologous with the central nervous system of a chordate. Also, the enteropneust dorsal nervous system is not associated with a notochord or neurenteric canal. The dorsal nervous system of enteropneusts resembles the ectoneural nervous system of echinoderms in being intra-epidermal and centred in the mesosome, the left half of which is homologous with the echinoderm water vascular system.

\section{R. P. S. Jefferies}

Department of Palaeontology,

The Natural History Museum,

London SW7 5BD, UK

\section{N. A. Brown}

MRC Experimental Embryology and Teratology Unit,

St George's Hospital Medical School, London SW17 ORE, UK

\footnotetext{
1. Arendt, D. \& Nübler-Jung, K. Nature, 371, 26 (1994).

2. Geoffroy de St Hilaire, E. Mém. Mus. Hist. nat. 9, 89-119 (1822).

3. Jefferies, R. P. S. The Ancestry of Vertebrates (British Museum (Natural History) \& Cambridge Univ. Press, 1986).

4. Jefferies, R. P. S. Ciba Fdn Symp. 162, 94-127 (1991)

5. Jefferies, R. P. S. Palaeontology 33, 631-679 (1990).

6. Jefferies, R. P. S., Brown, N. A. \& Daley, P. E. J. Acta zool., Stockh. (submitted).

Lacalli, T. C. Nature 373, 111 (1995)

8. Peterson, K. J. Nature 373, 112 (1995).
} 